\title{
Design of liposomal formulations for cell targeting
}

\author{
Eugénia Nogueira ${ }^{\mathrm{a}, \mathrm{b}}$, Andreia C. Gomes ${ }^{\mathrm{a}}$, Ana Preto ${ }^{\mathrm{a}}$, Artur Cavaco-Paulo ${ }^{\mathrm{b}, *}$ \\ a CBMA-Centre of Molecular and Environmental Biology, Department of Biology, University of Minho, Campus of Gualtar, 4710-057 Braga, Portugal \\ ${ }^{\mathrm{b}}$ CEB-Centre of Biological Engineering, University of Minho, Campus of Gualtar, 4710-057 Braga, Portugal
}

\section{A R T I C L E I N F O}

\section{Article history:}

Received 22 July 2015

Received in revised form

16 September 2015

Accepted 18 September 2015

Available online 25 September 2015

\section{Keywords:}

Drug delivery

Ligand

Liposome characterization

Liposome preparation

Targeting

Stealth

\begin{abstract}
A B S T R A C T
Liposomes have gained extensive attention as carriers for a wide range of drugs due to being both nontoxic and biodegradable as they are composed of substances naturally occurring in biological membranes. Active targeting for cells has explored specific modification of the liposome surface by functionalizing it with specific targeting ligands in order to increase accumulation and intracellular uptake into target cells. None of the Food and Drug Administration-licensed liposomes or lipid nanoparticles are coated with ligands or target moieties to delivery for homing drugs to target tissues, cells or subcellular organelles. Targeted therapies (with or without controlled drug release) are an emerging and relevant research area. Despite of the numerous liposomes reviews published in the last decades, this area is in constant development. Updates urgently needed to integrate new advances in targeted liposomes research. This review highlights the evolution of liposomes from passive to active targeting and challenges in the development of targeted liposomes for specific therapies.
\end{abstract}

(C) 2015 Elsevier B.V. All rights reserved.

\section{Contents}

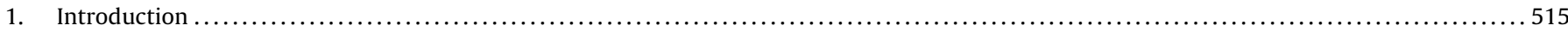

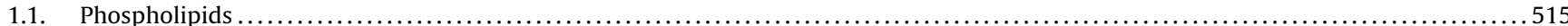

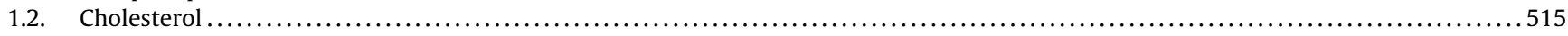

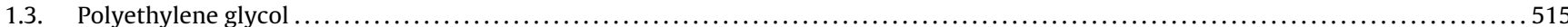

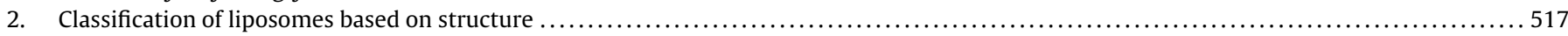

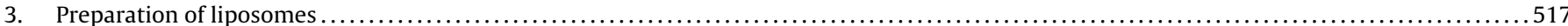

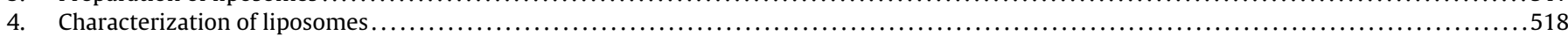

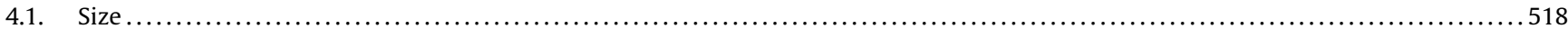

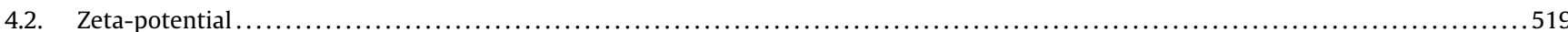

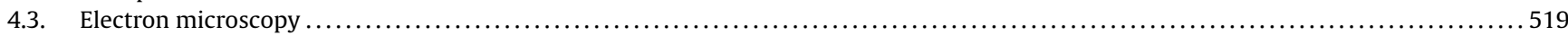

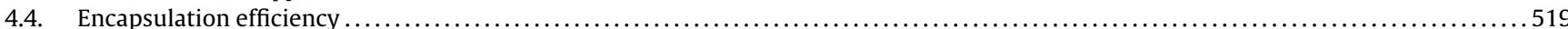

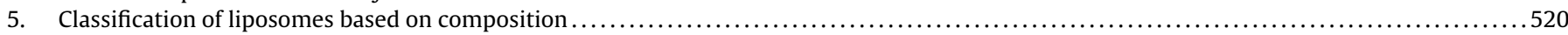

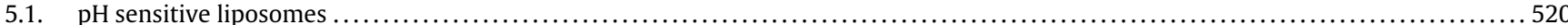

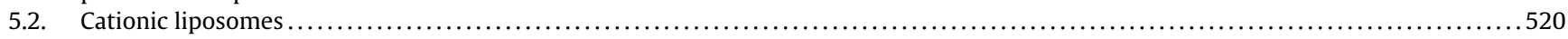

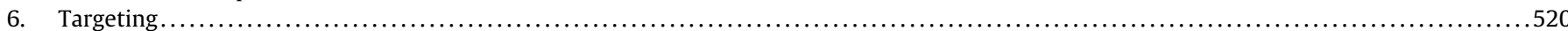

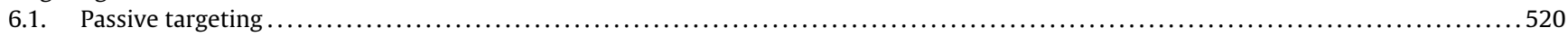

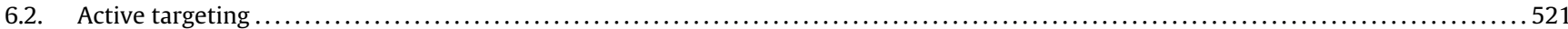

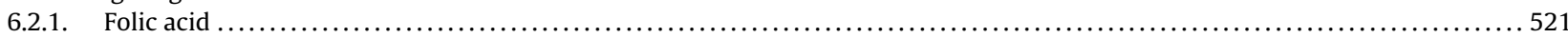

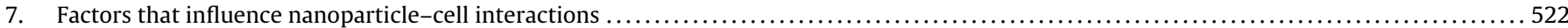

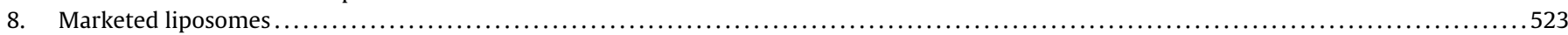

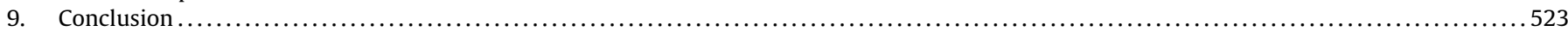

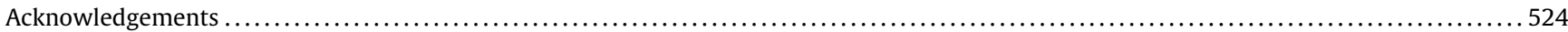

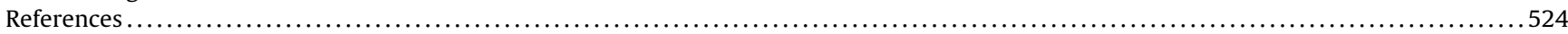

\footnotetext{
* Corresponding author. Fax: +351 253604429.

E-mail address: artur@deb.uminho.pt (A. Cavaco-Paulo).
} 


\section{Introduction}

Liposomes were first discovered in the late 1960s by the British hematologist Dr. Banghan [1] and Horne when testing a new electron microscope with a negative stain to dry phospholipids [2]. The resemblance between the captured micrographies and the plasmalemma served as the first evidence for the bilayer lipid structure of cell membranes [3]. In the following years, a variety of enclosed phospholipid bilayer structures consisting of single bilayers, initially termed 'bangosomes' and then 'liposomes' [4], were described. Early pioneers such as Gregory Gregoriadis, established the concept that liposomes could encapsulate drugs and thus be used as drug delivery systems $[5,6]$.

Liposomes were defined as artificial microscopic vesicles consisting of an aqueous core surrounded by one or more concentric phospholipid layers (lamellas) [7]. Liposomes showed a wide range of potential application as they are able to incorporate hydrophilic (in the aqueous compartment), hydrophobic (within lipidic membrane) and amphiphilic substances (lipid aqueous interface) [8]. For all these reasons, liposomes are the most popular nanocarrier systems used since they were discover.

Being both nontoxic and biodegradable, liposomes have gained extensive attention as carriers for a several therapeutic agents as they are composed of naturally occurring components. Biologically active materials encapsulated into the liposomes are protected from immediate dilution or degradation. The unique ability of liposomes to entrap drugs both in the aqueous and in the lipid phase make them attractive delivery systems for hydrophilic and hydrophobic drugs (Fig. 1a) [9].

In liposome formation, dissolved lipid molecules, consisting of a hydrophilic head group and a hydrophobic tail, self-assemble into bimolecular lipid leaflets upon reduction of their solubility in the surrounding medium. In contrast to common amphiphiles, that have critical micelle concentrations (CMC) of $10^{-2}-10^{-4} \mathrm{M}$, the CMC of bilayer forming lipids is four to five orders of magnitude smaller, meaning that the water solubility of these materials is extremely low [10]. When the lipids are exposed to an aqueous environment, interactions between them (hydrophilic interactions between polar head groups and van der Waals interactions between hydrocarbon chains and hydrogen bonding with water molecules) lead to spontaneous formation of closed bilayers, involved within a certain volume of solvent [11]. These structures may consist of one or several concentric membranes, varying in size from $20 \mathrm{~nm}$ to few $\mu \mathrm{m}$ in diameter, with a membrane thickness of approximately 4-5 nm [12,13].

\subsection{Phospholipids}

The lipids used to produce the liposomes may be changed or modified to customize the properties of the liposomal surface and membrane layer [14]. The most commonly used lipids are phospholipids, the major structural components of biological membranes. They are amphipathic molecules in which a glycerol bridge links a pair of hydrophobic chains and a hydrophilic polar head group, containing a phosphate group and a simple organic molecule [15]. One exception to this rule is sphingomyelin, which is derived from sphingosine instead of glycerol. The hydrophobic part consists of two hydrocarbon chains of saturated or unsaturated fatty acids, which esterify the same number of hydroxyl groups of glycerol, and each chain can present 10-28 carbon atoms. The nature of the fatty acid residues in each lipid molecule, namelly the number of double bonds in the chain, is responsible for fundamental bilayer properties such as elasticity and phase behavior [10]. The addition of organic molecules to the phosphate head group creates a variety of phospholipid species such as phosphotidylethanolamine
(PE), phosphotidylserine (PS), phosphatidyglycerol (PG) and phosphatidylcholine (PC) (Table 1) [16].

Phospholipids are abundant in nature and certainly those containing choline are the most used in the preparation of liposomes [15]. These are called phosphatidylcholine or lecithin (of the "lekithos" a Greek word meaning egg yolk) and are dipolar molecules at physiological $\mathrm{pH}$, with the quaternary ammonium group presenting a positive charge and a negative charge in the phosphate group [17].

Each class of phospholipid comprises a wide variety of molecules, concerning the type of fatty acids linked to the glycerol skeleton. Lecithin, as well as the other phosphatidic acids, can also be obtained by synthesis, presenting a well-defined composition, particularly with respect to acyl chains, which can be obtained from saturated or unsaturated hydrocarbon chains, with more or less number of carbon atoms [13]. These type of phospholipids can be easily characterized for various thermodynamic parameters, in comparison to the heterogeneous populations in natural phospholipids.

\subsection{Cholesterol}

Liposomes may suffer chemical and physical alterations, which shorten their shelf-life, limiting drug stability and storage conditions. This physical instability leads to problems of high permeability which induce undesired or too fast leakage rates [17]. To overcome these issues, the manipulation of lipid membrane components constitute the first attempt to modify bilayer fluidity. The addition of sterols is often required to modulate membrane rigidity and stability [18]. Sterols are vital constituents of natural membranes, playing a critical role in the regulation of membrane fluidity. Cholesterol is the most common sterol in mammalian membranes, being present in several liposomal based therapies approved by Food and Drug Administration (FDA) including DaunoXome ${ }^{\circledR}$, Myocet ${ }^{\circledR}$, Depocyt ${ }^{\circledR}$, Marqibo ${ }^{\circledR}$ and Doxil ${ }^{\circledR}[19]$. The cholesterol molecules are phospholipids with a tricyclic ring inserted between the first few carbons of the fatty acyl chains, into the hydrocarbon core of the bilayer, and the hydroxyl group facing the water phase [15].

Cholesterol reduces the permeability of water soluble molecules through the membrane, improves the fluidity as well as the stability of bilayer membrane in the presence of biological fluids such as blood/plasma [8]. Indeed, cholesterol prevents the interaction and destabilization of liposomes by blood proteins such as albumin, $m$-transferrin and macroglobulin [15]. The reduction of this interaction with blood proteins results in decreased binding of plasma opsonins responsible for rapid clearance of liposomes from the systemic circulation [20]. Variations in cholesterol composition can have profound effects on liposome formation, membrane stability and permeability. Incorporation of cholesterol into liposomes at $30 \mathrm{~mol} \%$ eliminates the phase transition of diacylphospholipids, reduces membrane permeability and forces the bilayer into a stable gel-like state. Below $30 \mathrm{~mol} \%$ cholesterol does not pack uniformly in diacylphospholipids and fails to completely eliminate their phase transition, while above $50 \mathrm{~mol} \%$, cholesterol phase separates in the membrane and can form crystals [19].

\subsection{Polyethylene glycol}

Unmodified nanoparticles do not survive long in circulation, being recognized by macrophages of the reticuloendothelial system (RES) within a few hours after administration [21]. The main sites of nanoparticle clearance are liver and spleen, where macrophages are in direct contact with the bloodstream [22]. Although several approaches for design and engineering of long circulating nanoparticles have been described, surface stabilization of nanoparticles 


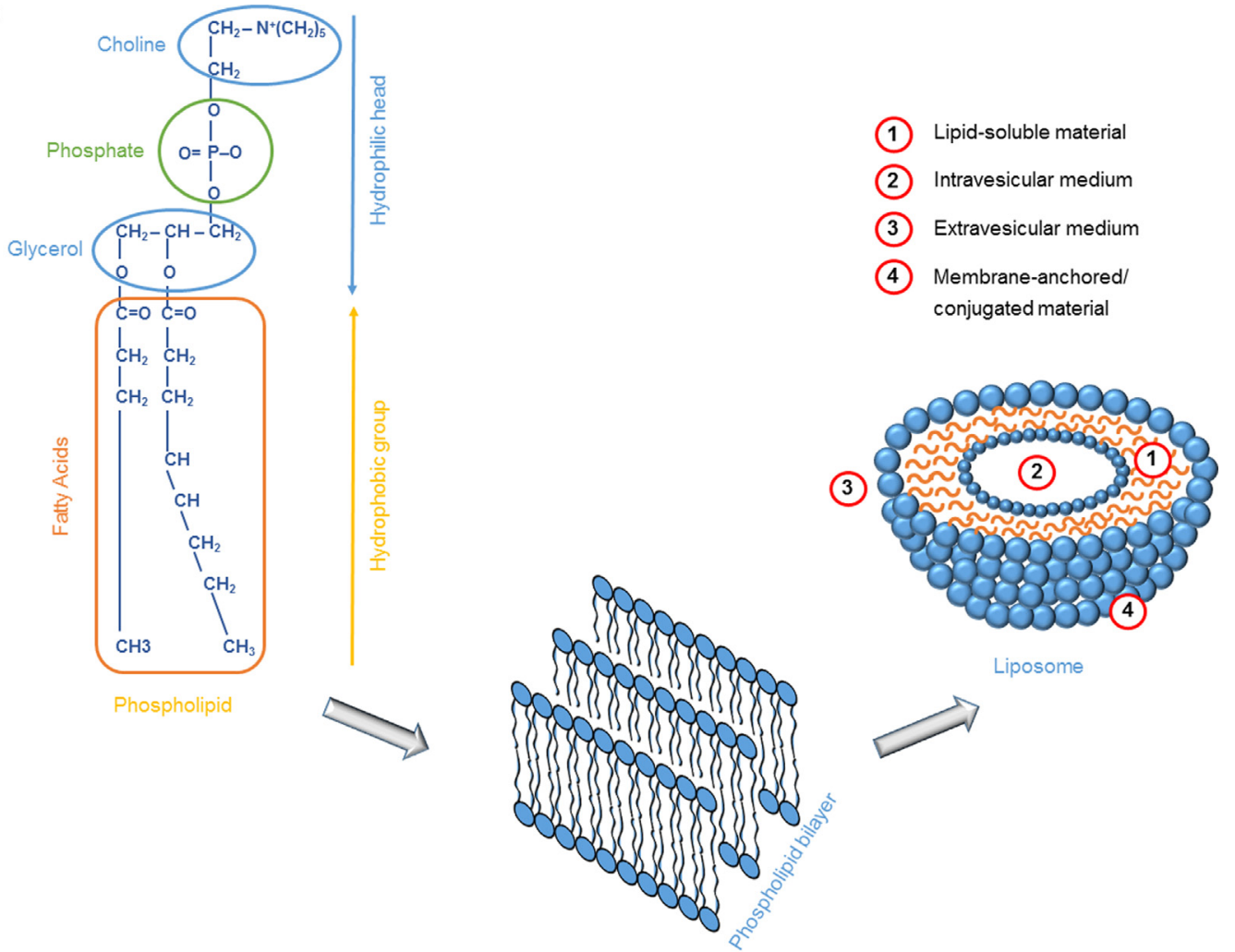

b

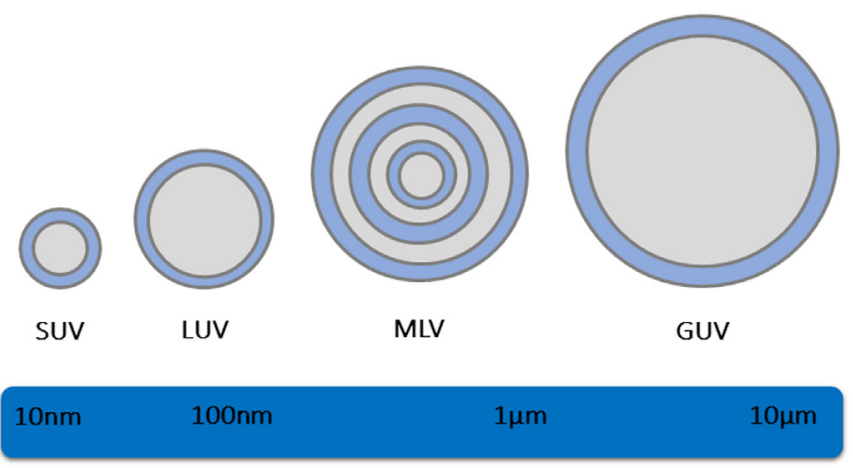

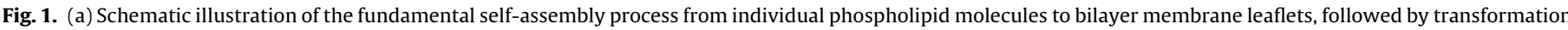
into liposomes. (b) Schematic representation of the different types of liposomes classified according to the size and number of lipid bilayers.

Table 1

Examples of phospholipids.

\begin{tabular}{|c|c|c|c|}
\hline Phosphatidic acid & Abbreviation & Derivate synthetic phospholipid & Abbreviation \\
\hline Phosphatidylcholine & PC & 1,2-Dimyristoyl-sn-glycero-3-phosphocholine & DMPC \\
\hline \multirow{3}{*}{$-\mathrm{CH}_{2}-\mathrm{CH}_{2}-\mathrm{N}\left(\mathrm{CH}_{3}\right)_{3}+$} & & 1,2-Dioleoyl-sn-glycero-3-phosphocholine & DOPC \\
\hline & & 1,2-Dipalmitoyl-sn-glycero-3-phosphocholine & DPPC \\
\hline & & 1,2-Distearoyl-sn-glycero-3-phosphocholine & DSPC \\
\hline \multirow{4}{*}{$\begin{array}{l}\text { Phosphotidyl- } \\
\text { ethanolamine } \\
-\mathrm{CH}_{2}-\mathrm{CH}_{2}-\mathrm{NH}_{3}+\end{array}$} & PE & 1,2-Dimyristoyl-sn-glycero-3-phosphoethanolamine & DMPE \\
\hline & & 1,2-Dioleoyl-sn-glycero-3-phosphoethanolamine & DOPE \\
\hline & & 1,2-Dipalmitoyl-sn-glycero-3-phosphoethanolamine & DPPE \\
\hline & & 1,2-Distearoyl-sn-glycero-3-phosphoethanolamine & DSPE \\
\hline Phosphotidylserine & PS & 1,2-Dimyristoyl-sn-glycero-3-phosphoserine & DMPS \\
\hline \multirow[t]{3}{*}{$-\mathrm{CH}_{2} \mathrm{CH}-\mathrm{NH}_{2} \mathrm{COOH}$} & & 1,2-Dioleoyl-sn-glycero-3-phosphoserine & DOPS \\
\hline & & 1,2-Dipalmitoyl-sn-glycero-3-phosphoserine & DPPS \\
\hline & & 1,2-Distearoyl-sn-glycero-3-phosphoserine & DSPS \\
\hline Phosphatidyglycerol & PG & 1,2-Dimyristoyl-sn-glycero-3-phosphoglycerol & DMPG \\
\hline \multirow{3}{*}{$-\mathrm{CH}_{2}-\mathrm{CHOH}-\mathrm{CH}_{2} \mathrm{OH}$} & & 1,2-Dioleoyl-sn-glycero-3-phosphoglycerol & DOPG \\
\hline & & 1,2-Dipalmitoyl-sn-glycero-3-phosphoglycerol & DPPG \\
\hline & & 1,2-Distearoyl-sn-glycero-3-phosphoglycerol & DSPG \\
\hline
\end{tabular}


with a range of nonionic surfactants or polymeric macromolecules has proved to be one of the most successful [23,24]. The unique physical properties make polyethylene glycol (PEG) commonly used to improve the stability and biological performance of colloidal drug carriers. The grafting of PEG to the surface of liposomes clearly extends its circulation lifetime [21]. This ability of PEG has been attributed mostly to its physical properties such as unlimited water solubility, large excluded volume and high degree of conformational entropy [25]. Some authors also support the reduction or prevention of protein adsorption [26] as well as a key role of PEG steric barrier that prevents aggregation of colloidal carriers and thus enhances their stability in vivo [27]. More recently, some studies have suggested a "dysopsonization" phenomenon where PEG actually promotes binding of certain proteins that then act to mask the vehicle [28]. We recently demonstrated a reduction in internalization of PEGylated nanoparticles by activated RES macrophages [29]. We showed that by increasing PEG concentration from 5 to $10 \%$, the stealth degree of nanoparticles clearly improved, as the internalization of nanoparticles by macrophages is greatly reduced, which is consistent with the current scaling models for polymers at interfaces. These models predict a mushroom-brush transition in PEG conformation at 5\% of PEG-lipid, when PEG coils start to repel each other and extend out from the surface on to which they are grafted [30]. If the polymer density is low it is said to be in the mushroom regime, when the graft density is high the polymers are said to be in the brush regime [31].

Conversely, recent reports demonstrate that repeated injections of PEGylated liposomes in the same animals at certain intervals induces significant immune responses, resulting in a loss of longcirculating properties [32,33]. This effect is called the "accelerated blood clearance $(\mathrm{ABC})$ " phenomenon. Ishida and coworkers suggests that anti-PEG IgM, produced by the spleen in response to the first dose, selectively binds to the PEG chains on a second dose administered several days later, and subsequently activates the complement system, one of the major opsonins, and enhances hepatic uptake of the subsequent doses [34,35]. The occurrence and the magnitude of the $A B C$ induction by liposomes are influenced by the dose and physicochemical properties of the PEGylated liposomes, the time interval between repeated injections, and by the species of the encapsulated drugs [36]. However, many approaches have been applied to minimize the immunogenicity of the PEG moiety upon repeated administration. It has been reported that PEG-lipids with a shorter alkyl chain could dissociate more rapidly from the lipid bilayer than conventional PEG-lipids, such as MPEG-DSPE and mPEG-CH, attenuating the occurrence of the $A B C$ phenomenon $[37,38]$.

\section{Classification of liposomes based on structure}

The size of liposomes vary from very small $(0.025 \mu \mathrm{m})$ to large $(2.5 \mu \mathrm{m})$ vesicles, showing one or several bilayer membranes. Vesicle size is a critical factor in determining the circulation half-life of liposomes and, together with the number of bilayers, affects the amount of drug encapsulation in the liposomes. According to their size and number of bilayers, liposomes can also be classified into one of two categories: (1) multilamellar vesicles (MLV) and (2) unilamellar vesicles. Unilamellar vesicles can also be classified into three categories: (1) giant unilamellar vesicles (GUV), (2) large unilamellar vesicles (LUV) and (3) small unilamellar vesicles (SUV) (Fig. 1b) [8]. While in unilamellar liposomes the vesicle has a single phospholipid bilayer sphere enclosing the aqueous solution, in multilamellar liposomes it has an onion like structure. Typically, several unilamellar vesicles will form on the inside of the other, building a multilamellar structure of concentric phospholipid spheres separated by layers of water [39].

\section{Preparation of liposomes}

The widespread use of liposomes for several purposes has created the need to develop preparation methods which should be efficient, reproducible and with the greatest simplicity possible. There are different methods available for preparation of liposomes, with numerous variants. Because of its simplicity, most laboratory use the lipid thin-film hydration method, first described in 1965 [40]. This method involves drying a solution of lipids, forming a thin film at the bottom of round-bottom flask and then hydrating the film with an aqueous buffer and vortexing the dispersion for some time [41]. The temperature of the hydrating medium should be above the gel liquid crystal transition temperature of the lipid, which should be maintained during the hydration period [42]. The compounds to be encapsulated are added either to an aqueous buffer or to an organic solvent containing lipids, depending on their solubility [43]. To reduce particle size and form unilamellar vesicle, sonication, homogenization or extrusion is applied.

Disruption of MLV suspensions by sonic energy (sonication) normally produces SUV, with diameters in the range of $15-50 \mathrm{~nm}$. The commonest equipment for preparation of sonicated particles are bath and probe tip sonicators [44]. Homogenization techniques depend on high-velocity collisions to reduce particle size. Mayhew et al. [45] have developed a microemulsifier that splits a sample of large, heterogeneous lipid particles into two streams and recombine them in a continuous, multicycle, high-velocity, high shear-force collision, producing monodisperse liposomes with a diameter of less than $100 \mathrm{~nm}$ [3]. In membrane extrusion, size is reduced by passing the liposomes through a membrane filter of defined pore size. The nucleation track type filter is composed of thin continuous sheet of polycarbonate, consisting of straight pore holes of exact diameter offering less resistance to liposomes passage [43].

As an alternative to the thin-film hydration method, liposomes can be produced by mixing the organic phase containing the dissolved lipid with the aqueous phase. Reverse-phase evaporation methods are based on this strategy, creating inverted micelles. The slow elimination of the organic phase leads to the disruption of these inverted micelles and consequent formation of liposomes [39]. An alternative approach is the rapid injection of lipids dissolved in organic solvent into an excess of aqueous solution. First described in 1973 by Batzri and Korn [46], the ethanol injection method ensures a narrow distribution of small liposomes (under $100 \mathrm{~nm}$ ), by simply injecting an ethanolic lipid solution in water, without the need of any size reduction method [8].

Detergent dialysis or depletion procedure can be used as an alternative for the manipulation of agents sensitive to denaturation, such as some proteins and oligonucleotides. Lipids are mixed with a surfactant or detergent in aqueous solution to produce micelles, and subsequent dilution or removal of the detergent result in liposomes encapsulating proteins and oligonucleotides in their native form [3].

There are other methods described for liposome preparation, including double emulsion, freeze-thaw, dehydrationrehydration, fast-extrusion and, recently, the use of supercritical carbon dioxide. In this procedure, a phospholipid solution is sprayed through supercritical carbon dioxide, causing the rapid evaporation of the solvent [47]. This leads to phospholipid precipitation as empty vesicles that can then be rehydrated using a range of aqueous solutions.

In summary, although there are several methods of liposome preparation, scale-up issues should be considered early in the development of new formulations for pharmaceutical application. Relevant advantages and disadvantages of the techniques discussed above are summarized in Table 2 . 
Table 2

Select methods of liposome preparations and their advantages in scale-up procedures (adaptated from Kraft et al.) [3].

\begin{tabular}{|c|c|c|}
\hline Method & Basic technique & Advantage for scaling \\
\hline \multicolumn{3}{|l|}{ Formulation method } \\
\hline Thin film hydration & Solvent evaporation followed by rehydration in aqueous phase & Simple \\
\hline Reverse-phase evaporation & Mixing of immiscible solvent with aqueous phase to form emulsion followed by evaporation of solvent & Simple \\
\hline Solvent injection & Injection of miscible solvent (generally ethanol) into aqueous phase & $\begin{array}{l}\text { Single-step process } \\
\text { Continuous processing }\end{array}$ \\
\hline Detergent depletion (dialysis) & Mixed-micelle formation with detergent followed by detergent dilution or removal & Gentle \\
\hline Supercritical fluid & $\begin{array}{l}\text { Solvation of lipids in supercritical carbon dioxide followed by injection } \\
\text { into low-pressure aqueous phase }\end{array}$ & $\begin{array}{l}\text { No organic solvent } \\
\text { Sterility }\end{array}$ \\
\hline \multicolumn{3}{|l|}{ Size reduction } \\
\hline Sonication & Ultrasonic energy to disrupt vesicles & Simple \\
\hline $\begin{array}{l}\text { High-pressure } \\
\text { homogenization }\end{array}$ & High-velocity collisions mechanically disrupt vesicles & $\begin{array}{l}\text { Monodisperse population } \\
\text { Reproducible } \\
\text { Continuous processing }\end{array}$ \\
\hline Membrane extrusion & Forcing through a filter of defined pore size & $\begin{array}{l}\text { Monodisperse population } \\
\text { Reproducible } \\
\text { Continuous processing }\end{array}$ \\
\hline
\end{tabular}

\section{Characterization of liposomes}

The performance of liposomes as drug delivery systems is strongly related to their structure at supramolecular, microscopic and nanoscopic level. The characterization of the structural vesicles is relevant for understanding the performance of these devices [17]. It is vital to assess the liposome quality and to obtain quantitative measures that allow comparison between different batches of liposomes [8]. The next section aims to highlight the importance of some structural parameters of vesicular systems, and present analytical techniques used to measure them.

\subsection{Size}

The range and vesicle size distribution gives an idea of the size homogeneity of vesicles forming the same system. These are therefore essential parameters for a good characterization of a vesicular formulation [17]. It is known that size influences pharmacokinetics, tissue distribution and clearance of liposomes. Indeed, hepatic uptake and accumulation, tissue extravasation, tissue diffusion and kidney excretion is deeply dependent of particle size. In contrast to small liposomes, that exit or enter fenestrated vessels in the liver endothelium or tumor microenvironment, bigger liposomes are taken up by phagocytes or remain in the tissues for an extended time. Thereafter, the phagocytes with liposomes accumulate in the spleen and liver for eventual elimination. Furthermore, cellular internalization pathways may also be influenced by particle size [3].

Several techniques are available for evaluating the size and size distribution of liposomes, including microscopy techniques, size-exclusion chromatography (SEC), field-flow fractionation and static or dynamic light scattering (DLS) [8]. Among them, DLS, also referred to as photon correlation spectroscopy or quasi-elastic light scattering, represents a fast technique for size measurements. DLS is an analysis of time dependence of intensity fluctuation in scattered laser light due to Brownian motion of particles in solution. Small particles diffuse more rapidly than large particles and the rate of fluctuation of scattered light intensity varies accordingly. Thus, the translational diffusion coefficient $(D)$ can be measured, which in turn can be used to determine the mean hydrodynamic radius $\left(r_{\mathrm{h}}\right)$ of particles using Stoke-Einstein equation (Eq. (1)), where $k$ is the Boltzmann constant, $T$ is the temperature, and $\mathrm{g}$ is the solvent viscosity [43].

$$
r_{\mathrm{h}}=\frac{K T}{6 \pi \eta D}
$$

Table 3

Liposomes classification based on composition and mode of drug delivery (adapted from Sharma et al.) [60].

\begin{tabular}{|c|c|c|}
\hline Type & Composition & Characteristics \\
\hline $\begin{array}{l}\text { Conventional } \\
\text { liposomes }\end{array}$ & $\begin{array}{l}\text { Neutral and/or negatively charged } \\
\text { phospholipids and cholesterol }\end{array}$ & $\begin{array}{l}\text { Subject to coated pit endocytosis contents ultimately } \\
\text { delivered to lysosomes if they do not fuse from the } \\
\text { endosome, useful for RES targeting }\end{array}$ \\
\hline pH sensitive liposomes & Phospholipid such as PE, DOPE & $\begin{array}{l}\text { Subject to coated pit endocytosis at low } \mathrm{pH} \text { fuse with cell } \\
\text { or endosome membrane and release their contents in the } \\
\text { cytoplasm }\end{array}$ \\
\hline Cationic liposomes & Cationic lipids & $\begin{array}{l}\text { Possibly fuse with cell or endosome membrane; suitable } \\
\text { for delivery of negatively charged macromolecules (DNA, } \\
\text { RNA); ease of formation; toxic at high dose }\end{array}$ \\
\hline $\begin{array}{l}\text { Long circulating } \\
\text { liposomes or stealth } \\
\text { liposomes }\end{array}$ & $\begin{array}{l}\text { Neutral high transition temperature; lipid, } \\
\text { cholesterol and } 5-10 \% \text { of PEG-DSPE }\end{array}$ & $\begin{array}{l}\text { Hydrophilic surface coating; low opsonisation and thus } \\
\text { low uptake by RES; long circulating half life }\end{array}$ \\
\hline Immunoliposomes & $\begin{array}{l}\text { Conventional or long circulating liposomes } \\
\text { with attached monoclonal antibody or } \\
\text { recognition sequence }\end{array}$ & $\begin{array}{l}\text { Subject to receptor mediated endocytosis; cell specific } \\
\text { binding; can release contents extracellularly near the } \\
\text { target tissue and drug diffuse through plasma membrane } \\
\text { to produce their effects }\end{array}$ \\
\hline Magnetic liposomes & $\begin{array}{l}\text { PC, cholesterol, small amounts of linear chain } \\
\text { aldehyde and colloidal particles of magnetic } \\
\text { iron oxide }\end{array}$ & $\begin{array}{l}\text { Liposomes that indigenously contain binding sites for } \\
\text { attaching other molecules like antibodies on their exterior } \\
\text { surface. Can be made use of by an external magnetic field } \\
\text { in their deliberate, on site and rupture immediately release } \\
\text { of their contents }\end{array}$ \\
\hline $\begin{array}{l}\text { Temperature or heat } \\
\text { sensitive liposomes }\end{array}$ & DPPC & $\begin{array}{l}\text { Vesicles shows maximum release at } 41^{\circ} \mathrm{C} \text {.The phase } \\
\text { transition temperature of DPPC liposomes released the } \\
\text { entrapped contents at the target cell surface }\end{array}$ \\
\hline
\end{tabular}


Determination of particle diameter using Stokes-Einstein equation.

The value of polydispersity index (PDI), indicative of the degree of heterogeneity regarding sample size, is another parameter possible to calculate. Size distribution can be monodisperse (narrow distribution) or polydisperse (broad distribution). The calculation of PDI takes into account the particle mean size, the refractive index of the solvent, the measurement angle and the variance of the distribution [48]. If a scale from 0 to 1 is considered, a PDI lower than 0.1 indicates a high homogeneity of the particle population, whereas high PI values suggest a broad size distribution or even several populations [49].

A new methodology for the characterization of vesicular systems was recently developed: the nanoparticle tracking analysis (NTA) [17]. This technique allows a direct and real-time visualization and analysis of nanoparticles in liquids, by combining laser light scattering microscopy with a charge-coupled device camera [50]. During NTA analysis, a laser beam illuminates the particles in suspension and the light scattered by each particle is focused by the microscope onto the image sensor of the video camera. The NTA software identifies and tracks each particle, thus enabling measurement of the mean square displacement of particle movement. This parameter, together with the temperature and the viscosity of the liquid containing the particle, is used to calculate particle size through the Stokes-Einstein equation [51]. The capacity of NTA to simultaneously measure size and particle scattering intensity, besides allowing to distinguish particles of different refractive index within the same sample solution, makes a direct estimation of particle concentration [17].

\subsection{Zeta-potential}

The nature and density of the surface charge of liposomes may influence stability, biodistribution, pharmacokinetics, and cellular affinity and drug internalization. The net charge may vary from negative, neutral or positive, depending on the lipid composition and the head group of lipids. The overall net charge of the vesicles is classically expressed as surface or zeta potential [3]. In this way, zeta potential is a physical property characteristic of all particles in suspension. The particles dispersed in an aqueous medium will acquire a surface charge, by ionization of surface groups or adsorption of charged species. These ions create a layer surrounding the particle surface, commonly named the Stern layer. Another layer outside the Stern layer is also formed, where ions diffuse more freely [52]. Within the diffuse layer, there is a notional boundary where the ions and particles form a stable entity, and when a particle moves, ions within the boundary move accordingly. The zeta potential is the potential at the boundary (surface of hydrodynamic shear) beyond which ions stay with the bulk dispersant [17].

To measure the zeta potential, a laser beam passes through the center of the sample cell and the scattered light at an angle of about $13^{\circ}$ is detected. When an electric field is applied to the cell, any particles moving through the measurement volume will lead to fluctuation of the detected light with a frequency proportional to the particle speed [8]. The velocity of a particle in a unit electric field is referred to as its electrophoretic mobility. Zeta potential is related to the electrophoretic mobility by the Henry equation, (Eq. (2)) where, $U_{\mathrm{E}}$ is the electrophoretic mobility, the zeta $(\zeta)$-potential, $\epsilon$ is the dielectric constant, $f(\mathrm{Ka})$ is the role of Henry and $\eta$ is the viscosity of the medium [53].

$U_{\mathrm{E}}=\frac{2 \epsilon \zeta F(\mathrm{Ka})}{3 \eta}$

Determination of zeta-potential using the Henry equation.

\subsection{Electron microscopy}

Apart from size and surface properties, other parameters are vital for a proper characterization of vesicles, such as analysis of their morphology. Microscopy is the ideal tool for this purpose, since it allows the direct observation of the sample and evaluation of its morphological characteristics, such as shape and lamellarity [17]. The evolution of drug delivery systems to a nanometer scale lead to replacement of classical light microscopy by electron microscopy techniques, indispensable tools for the analysis of nanoemulsions. Indeed, the high resolution and methodological versatility of electron microscopy provide sufficient resolution for the visualization of nano-sized particles [54]. Several variants on electron microscopy (EM), such as transmission EM (TEM) and scanning EM (SEM), allows the visualization of each individual vesicle, to obtain precise information about the profile of liposome population(s) [43]. In TEM, a small amount of hydrated sample is placed in direct contact with the grid surface, which may affect their orientation or morphology before they are viewed. A negative stain, such as Uranyl Acetate or Osmium Tetroxide, is then used, resulting in the liposomes appearing as white against a black background [54-56]. In SEM, the sample is dried and its surface coated with a conductive layer, normally of gold. The structure is scanned point by point with the electron beam and secondary electrons that are emitted by the sample are detected. In this way, a three-dimensional (3-D) impression of the structures in the sample, or of their surface, is obtained [54,55]. In scanning transmission electron microscopy (STEM), TEMs are equipped with a scanner unit. This low-energy technique aims to overcome the charging effects of the nanoparticles, which may disturb normal SEM images that are obtained by analysis of secondary electrons with energies in the $\mathrm{eV}$ range [55]. EM techniques are very time consuming and require costly equipment, often inaccessible. Furthermore, sample preparation requires removal of liposomes from their native environment, which can generate artefacts, induce shrinkage and shape distortion. Attention should be given to sample preparation to overcome some of these problems and obtain reproducible and accurate results [8]. A new microscopy technique was developed to study liposome morphology, size and stability, the atomic force microscopy (AFM). This system allows the analysis of soft and biological materials, when it is possible to perform high-resolution imaging under conditions conducive, such liquid and without applying vacuum. In these conditions it is possible to evaluate the influence of temperature, addition of surfactant, ions, polymers, adsorption/desorption kinetics and biomolecules-membrane interactions [57].

\subsection{Encapsulation efficiency}

In addition to an extensive structural characterization, the capacity to encapsulate therapeutic agents is vital to evaluate the potential of vesicles as drug delivery systems. This ability can be expressed as encapsulation efficiency (EE), the percentage of drug encapsulated inside the vesicle aqueous core in relation to the initial amount of drug used for encapsulation (Eq. (3)). If it is not known where the drug is adsorbed/integrated in the vesicular membrane, it is more recommendable to use trapping or incorporation efficiencies [17].

$$
\begin{aligned}
& \text { Encapsulation efficiency (\%) } \\
& =\frac{\text { mass of encapsulated drug }}{\text { mass of drug }} \times 100
\end{aligned}
$$

Determination of the encapsulation efficiency of drugs.

As an alternative, capture (or encapsulation) volume is used for vesicular systems in which the cargo does not interact with 
the vesicular membrane. The entrapped volume of liposome (in $\mu \mathrm{L} / \mathrm{mg}$ phospholipids) is calculated from measurements of the total quantity of solute entrapped inside the liposome, assuring that the concentration of solute in the inner aqueous medium is the same after separation from non-encapsulated material $[2,58]$.

Classically, the quantification of encapsulated cargo requires the execution of the following steps: separation of the nonencapsulated cargo, disruption of the vesicular membrane, and quantification of the released material [17]. Size exclusion chromatography is a commonly used separation technique, based on the existing size difference between liposomes loaded with drug and the free drug. We might use dialysis membrane with an appropriate cut-off, or ultracentrifugation, a simple and fast method for the separation of drug-loaded liposomes from their medium. The lipidic bilayer can be disrupted with methanol or Triton X-100 and the released material is then quantifiable. Methods used for this quantification step depend on the nature of the cargo and include spectrophotometry, fluorescence spectroscopy, enzyme-based methods and electrochemical techniques [8].

\section{Classification of liposomes based on composition}

Liposomes have a key advantage in comparison with colloidal carrier systems: they allow innumerous alterations of their structural and physicochemical characteristics [59]. This flexibility possibilitates the modification of liposome behavior in vivo and their design according to specific therapeutic needs. In this way, it is possible to classify the possible liposome versions based on composition and in vivo application (Table 3).

\section{1. $p H$ sensitive liposomes}

PH-sensitive liposomes are stable at physiologic $\mathrm{pH}$ (7.4) but under acidic environments destabilize and acquire fusogenic characteristics, thus leading to the release of their aqueous contents. In the literature, several classes of $\mathrm{pH}$ sensitive liposomes, based on the mechanism of triggering $\mathrm{pH}$ sensitivity, are described. The best known $\mathrm{pH}$ sensitive component is PE and its derivatives with compound containing an acidic group that acts as a stabilizer at neutral $\mathrm{pH}$ [61]. In contrast to the majority of the phospholipids, the headgroup of PE is poorly hydrated and small, occupying a lower volume as compared to the respective hydrocarbon chains. In this way, to the contrary of cylinder shape of bilayerstabilizing phospholipids, PE presents a cone shape, which prevents the formation of a lamellar phase. The cone shape of PE molecules allows the creation of strong intermolecular interactions between the amine and phosphate groups of the polar headgroups [62]. Amphiphilic molecules containing a protonatable acidic group that is negatively charged at physiological $\mathrm{pH}$ can be incorporated alongside $\mathrm{PE}$ in the lipidic bilayer. These molecules allow the formation of bilayer structures and liposomes at physiological $\mathrm{pH}$ and temperature because, in addition to causing electrostatic repulsion between bilayers, they also disrupt the strong interactions between PE headgroups [63].

With this approach, stable liposomes are formed at physiological $\mathrm{pH}$ and, upon $\mathrm{pH}$ reduction, the carboxylic group of the amphiphiles reduces their stabilizing effect, converting the lipid membrane from a conventional bilayer sheet to inverted hexagonal phase [64].

\subsection{Cationic liposomes}

In contrast to neutral or negatively charged liposomes, cationic liposomes establish electrostatic interactions with negatively charged molecules such as nucleic acids, including plasmids, messenger RNAs and synthetic oligonucleotides, or proteins and peptides [65].

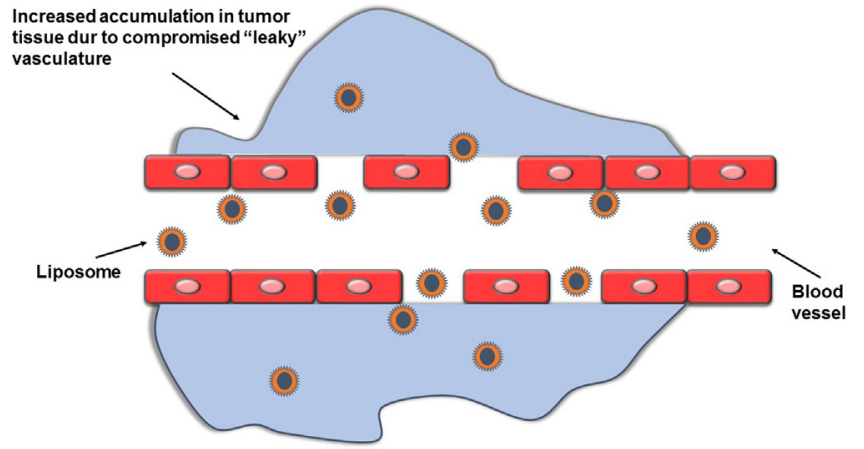

Fig. 2. Schematic illustration of passive targeting via the EPR effect.

Since the landmark publications in the late 1980s [66], cationic liposomes are one of the most efficient synthetic gene delivery reagents in vitro [67]. Felgner and colleagues demonstrated that when DNA is mixed with cationic liposomes it forms a cationic particle (lipoplex), able to protect DNA from enzymatic degradation and deliver it into cells by interacting with the negatively charged cell membrane [66]. Cationic lipids are positively charged amphiphilic molecules constituted by a cationic polar head group (typically amino groups), a hydrophobic domain (alkyl chains or cholesterol), and a linker linking the polar head group with the non-polar tail. They can form positively charged liposomes, usually in combination with a neutral helper lipid [65]. However, some disadvantages have been reported, representing limitations to their wider successful application. Cationic liposomes have limited delivery efficiency and gene silencing and toxicity at higher concentrations. Furthermore, adverse interactions may come from the presence of negatively charged macromolecules in serum and cell surfaces, and impaired ability to reach tissues beyond the vasculature unless directly injected into the tissue [68]. Toxicity may be associated to the large size of the complexes, and the high positive zeta potential necessary for their uptake. Indeed, the toxicity may results from the charge ratio between cationic lipid species and nucleic acids, as well as the high dose of lipoplexes administered [69].

\section{Targeting}

Specific targeting to the disease cell/tissue without affecting the healthy cells is the Holy Grail for clinical application. Targeting of tissues and cells can either be passive, for long circulating liposomes, or active, for liposomes conjugated to ligands with specific affinity for receptors within a tissue, cell or intracellular target [70].

\subsection{Passive targeting}

The knowledge that large particle potentiates uptake by phagocytic cells was the basis for the design of liposome-encapsulated amphotericin B. Enhanced drug accumulation in phagolysosomes is observed within the same phagocytes that engulfed the pathogenic fungi. Since phagocytes accumulate in the spleen, the antifungal drug amphotericin B can reach the intravesicular sites of fungal growth even without existing ligand-receptor interactions. This approach that exploits cellular and physiological pathways and the knowledge about particle clearance mechanisms is called passive targeting [3].

In the oncology field, passive targeting depends on the Enhanced Permeability and Retention effect (EPR). Due to abnormal leaky (new or neo) vasculature and impaired lymphatic drainage, small non targeted particles $(<400 \mathrm{~nm})$ circulating in the blood tend to accumulate in the interstitial space of tumors and inflamed tis- 
Table 4

Molecular targets and their targeting ligands.

\begin{tabular}{|c|c|c|c|c|}
\hline Type of ligand & Molecular target & Targeting ligand & Cell type & References \\
\hline \multirow[t]{2}{*}{ Antibodies } & mAb anti HER2 & HER2 & Breast cancer & {$[74-78]$} \\
\hline & mAb anti VEGF & VEGF & Angiogenesis in tumor environment & [79-83] \\
\hline \multirow{2}{*}{ Aptamers } & Aptamer against E-selectin & E-selectine & Inflamed vasculature in advanced tumors & {$[84,85]$} \\
\hline & Aptamer against nucleolin & Nucleolin & Breast cancer & [86-90] \\
\hline \multirow[t]{2}{*}{ Protein } & Transferrin & Transferrin receptor & Cancer cells & {$[91-100]$} \\
\hline & EGF & EGR receptor & Cancer cells & [101-105] \\
\hline \multirow[t]{2}{*}{ Peptides } & RGD peptide & ${ }_{\alpha} \mathrm{v} \beta 3$ integrin & Tumor neovasculature & [106-110] \\
\hline & Octreotide & SSTR2 & Ovarian cancer & [111-115] \\
\hline Carbohydrates & Galactose & ASGPR & Hepatocytes & [116-120] \\
\hline Vitamines & Folic acid & Folate receptor & Cancer cells, activated macrophages & [121-130] \\
\hline
\end{tabular}

sues, a known hallmark of cancer (Fig. 2) [3]. Fast growing tumor results in tissue anoxia and expression of growth factors like the vascular endothelial growth factor (VEGF), responsible by rapid angiogenesis. Newly formed blood vessels show a higher rate of vascular fenestration and consequent permeability in tumors. In this way, nanoparticles cross the walls of such blood vessels and can be retained in the tumor tissue [70].

However, the passive targeting effect is restricted to some tumors. Indeed, the EPR effect is only achieved in some solid tumors that are larger than approximately $4.6 \mathrm{~mm}$ in diameter. Moreover, the porosity and pore size of tumor vessels depends on the type and status of tumors [71]. The EPR effect is also affected by other factors, such as tumor location, the surrounding stroma, amount of infiltration by macrophages, patient characteristics such as age and gender, and additional medications [72]. Furthermore, nascent tumors and nonvascularized disease sites are unlikely to benefit from this EPR effect [3]. In passive targeting, prolonged blood circulation liposomes are particularly important, as to avoid their sequestration by macrophages of RES, and ensure their accumulation in tumor cells [70]. Due to the unpredictability of the EPR effect and their limitation to some tumors, a greater focus on cancer targeted therapy is imperative in the future.

\subsection{Active targeting}

In 1906, Lehner et al. introduced the concept of active targeting by describing a "magic bullet" needed to direct specific drug delivery within the body [70]. Active targeting exploits specific modification of liposomal surface with a targeting ligand, which can lead to their accumulation at the target site or intracellular delivery to target cells. The inclusion of certain ligands in liposomes allows the release of their contents intracellularly by receptor-mediated endocytosis. This mechanism, in addition to increasing the efficacy of the carried drug, may also overcome drug resistance at least partially by reducing their diffusion from the tumor [71].

The targeted molecule is typically selected based on its selectivity or overexpression in target cells. A number of targeting ligands have been proposed for development of targeted liposomal formulations. These include proteins (antibodies or antibody fragments), nucleic acids (aptamers) and other receptor ligands (peptides, carbohydrates, and vitamins) (Table 4). There are several aspects taken into account in the selection of target molecules, which include: relative degree of overexpression or selective expression on the target, target cell uptake of ligand-targeted formulation, and degree of covering of the target molecule [71,73].

Active targeting requires a targeting moiety to remain lipid associated in biologic environments. The crucial goal of targeted ligand conjugation is to bind a targeting moiety without losing its functionality after inclusion in the liposome [131]. Furthermore, it is also vital to ensure their stability in fluid flows, as well as the ease of preparation, scaling, and functional capacity to bind to target cells. If the targeting moiety is hydrophilic just can be stable in the membrane surface by conjugation to phospholipid or fatty acyl chains [3]. Otherwise, a hydrophobic anchor for a targeting moiety is only possible by chemical conjugation. Several procedures have been described in the literature with varying degrees of success and complexity (Fig. 3). The wide range of head groupmodified lipids commercially available has encouraged researchers to develop more complex liposomal formulations to meet clinical challenges [131]. The use of PEG-lipid conjugates for surface coating of liposomes is widespread. These conjugates are derived from mPEG, which has nonreactive methoxy terminal groups. To attach a targeting moiety to liposomes, previous approaches have been used to functionalize PEG molecules in PEG-lipid conjugates [71]. Furthermore, the broad spectrum of chemical approaches can also be used to conjugate therapeutic molecules, or contrast agents to liposome surface [131].

Typically, there are three main approaches available to functionalize liposomes. One approach is binding the desired targeting ligand to a lipid prior to mixing them with other lipid components during liposome preparation [132]. In another approach, immediately after preparation, liposomes are functionalized with the required targeting ligand [132]. Head group-modified lipids with a PEG spacer functionalized at the end with amine, carboxylic acid, thiol or maleimide groups represent available options for this approach [133]. Alternatively, the postinsertion of the functionalized lipid in preformed liposomes, was proposed. This method is based on the spontaneous incorporation of functionalized lipids from the micellar phase into preformed and even drug-loaded liposomes [134]. Derivatization of the targeting molecule happens in a separated step, this approach prevents the interference of activated lipids with other liposomal components such as those present in the buffer [134].

\subsubsection{Folic acid}

Folic acid is one of the most studied ligands in targeted drug delivery. Folic acid is a high affinity ligand for the folate receptor (FR), which remains even after derivatization through one of its carboxyl groups. FRs mediate cellular internalization of folate conjugates via receptor-mediated endocytosis being folate conjugation a method for targeted intracellular delivery of therapeutic agents to FR expressing cells [135]. Receptor-mediated endocytosis beginning with folate conjugates binding to a cell surface FR, which is succeeded by distinct steps [136]. After membrane invagination and internalization to form an endocytic vesicle, the $\mathrm{pH}$ of the vesicle lumen decreases by action of proton pumps localized in the endosomal membrane. This acidification allows the release of bound ligand and their delivery in to the cytosol, through a conformational change in the FR protein [137]. Finally, the FR recycles back to the cell surface, allowing for delivery of additional folate conjugates in to the target cell [136].

Apart from their high affinity for its receptor, folate has other unique properties being one attractive ligand for use in drug targeting. These features include its convenient availability, low molecular weight, facile conjugation chemistry, lack of immunogenicity, water solubility, stability to diverse solvents, $\mathrm{pH}$ and 

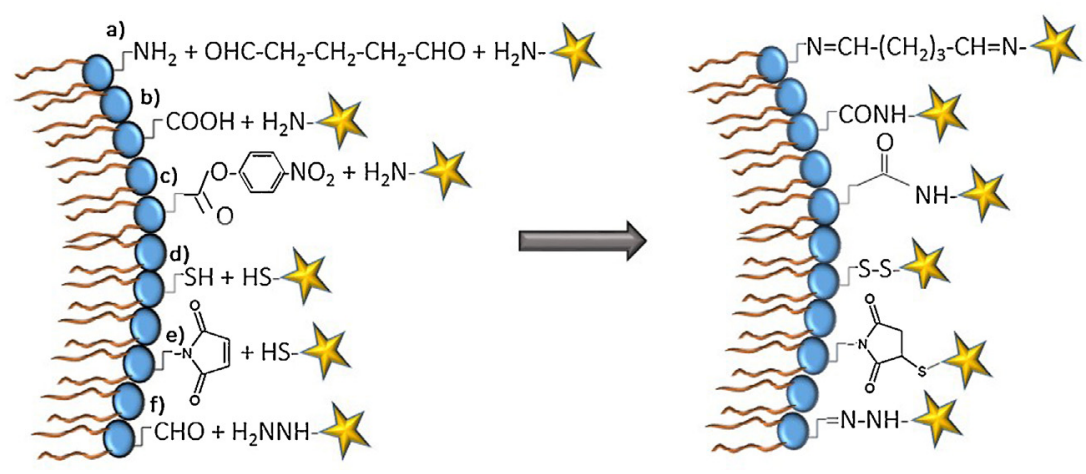

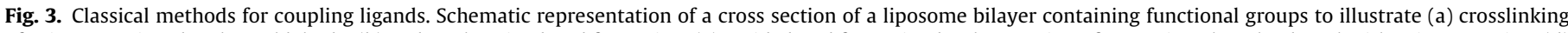

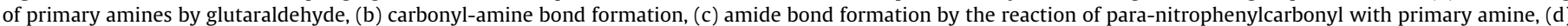

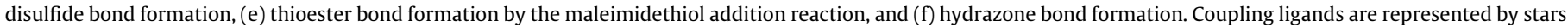
(adapted from Marqués-Gallego et al.) [132].

temperature. Furthermore, the small size of the folate ligand also allows good tissue penetration and rapid clearance from receptor negative tissues [136]. Therefore, a wide range of molecules and drug carriers have been conjugated to folate and evaluated for FR targeting [135].

A usual method to incorporate folate in a liposome coated with PEG polymers is to conjugate the ligand to the outer end of the PEG chain. Folate may be conjugated to amino-PEG through the gammacarboxyl to a phospholipid or cholesterol anchor and incorporated into the bilayer during liposome preparation. As described above, the affinity of the resulting conjugate to FR is about 10-fold lower than free folic acid. However, since liposome binding is mediated by several ligands, its overall affinity for a target cell is the product of the individual affinities. In this way, the affinity of folate conjugates for FR-expressing cells is much higher, and is needed nearly a 1000fold greater concentration of free folic acid to compete effectively with folate conjugates for FR binding [138]. Although these folate conjugates were shown to successfully target FR-overexpressing cancer cells, self-aggregation on the liposome surface has often been observed when using such formulations, leading to reduced FR targeting efficiency [139]. Furthermore, there are concerns over the two negative charges carried by folate-PEG-phospholipid and the use of a carbamate linker in folate-PEG-gholesterol, which has limited hydrolytic stability $[140,141]$.

\section{Factors that influence nanoparticle-cell interactions}

To produce nanoparticles that are capable of navigating the body and reach target cells, it is necessary to understand the physic- ochemical properties and their influence in the interaction with biological systems. Indeed, to design nanoparticles to accumulate in the target cell, it is crucial to understand the contribution of size, shape, and chemistry to this delivery process [142]. Only in this way it will be possible for researchers to develop complex nanoparticles that ensure the highest possible delivery efficiency while maximizing signal strength [143]. The next points describe some factors that influence nanoparticle-cell interactions (Fig. 4):

- Size: small nanoparticles have less ligand-to-receptor interaction than do larger nanoparticles.

- Shape: rods show the highest uptake, followed by spheres, cylinders, and cubes: greater membrane wrapping time required for the elongated particles.

- Charge: positively charged nanoparticles are taken up at a faster rate: the cell membrane possesses a slight negative charge and cell uptake is driven by electrostatic attractions.

- Ligand density: ligand's binding affinity increases proportionally to the size of a nanoparticle owing to a higher ligand density on the nanoparticle surface.

- Receptor expression levels: enough receptors with close proximity to one another to produce enough free energy to drive membrane wrapping.

- Internalization mechanism: each cell type can utilize different internalization pathways.

- Cell properties: because each cell type possesses a unique phenotype, optimal nanoparticle uptake size may depend on the cell being assayed.

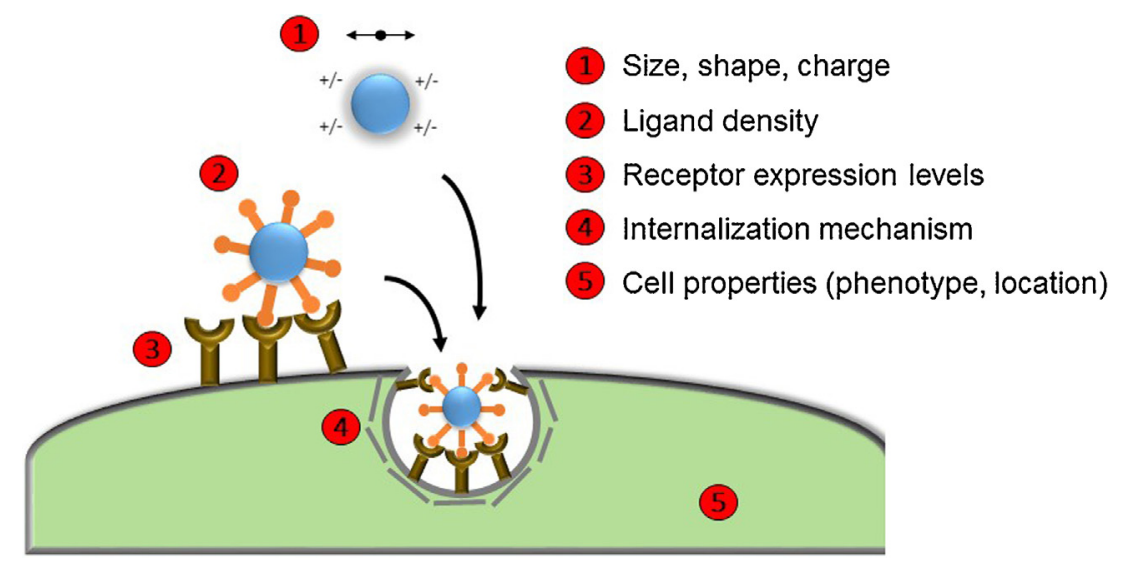

Fig. 4. List of factors that can influence nanoparticle-cell interactions at the nano-bio interface (adapted from Albanese et al.) [143]. 
Table 5

Marketed liposomal products.

\begin{tabular}{|c|c|c|c|c|}
\hline Trade name & Company & Drug & Indication & Route of administration \\
\hline \multicolumn{5}{|l|}{ Antifungal } \\
\hline Abelcet & Enzon & Amphotericin B & Invasive aspergillosis & Intravenous \\
\hline AmBisome & Gilead & Amphotericin B & Leishmaniasis & Intravenous \\
\hline Amphotec & Intermune & Amphotericin B & Invasive aspergillosis & Intravenous \\
\hline Amphocil & Three Rivers Pharmaceutical LLC & Amphotericin B & Invasive fungal & Intravenous \\
\hline \multicolumn{5}{|l|}{ Cancer therapy } \\
\hline DaunoXome & Galen & Daunorubicin & Kaposi's sarcoma & Intravenous \\
\hline Myocet & Cephalon & Doxorubicin & Breast cancer + cyclophosphamide & Intravenous \\
\hline Doxil, Caelyx & Johnson \& Johnson & Doxorubicin & $\begin{array}{l}\text { Kaposi's sarcoma, ovarian cancer, } \\
\text { breast cancer, multiple myeloma }\end{array}$ & Intravenous \\
\hline Lipo-Dox & Taiwan liposome & Doxorubicin & $\begin{array}{l}\text { Kaposi's sarcoma, breast and } \\
\text { ovarian cancer }\end{array}$ & Intravenous \\
\hline Marqibo & Talon & Vincristine & Acute lymphoblastic leukemia & Intravenous \\
\hline Onco TCS & INEX Pharmaceuticals & Vincristine & Non-Hodgkin’s Lymphoma & Intravenous \\
\hline Lipusu & Luye Pharma Group Ltd. & Paclitaxel & Solids tumors & Intravenous \\
\hline DepoCyt & Pacira & Cytarabine & $\begin{array}{l}\text { Lymphomatous, meningitis, } \\
\text { neoplastic, meningitis }\end{array}$ & Spinal \\
\hline \multicolumn{5}{|l|}{ Vaccine } \\
\hline Epaxal & Crucell & $\begin{array}{l}\text { Inactivated hepatitis A virus (strain } \\
\text { RG-SB) }\end{array}$ & Anti-viral: hepatitis A virus & Intramuscular \\
\hline Inflexal V & Crucell & $\begin{array}{l}\text { Inactivated hemaglutinine of } \\
\text { Influenza virus strains A and B }\end{array}$ & $\begin{array}{l}\text { Anti-viral: influenza virus strains A } \\
\text { and B }\end{array}$ & Intramuscular \\
\hline \multicolumn{5}{|l|}{ Analgesics } \\
\hline DepoDur & Pacira & Morphine & Postsurgical analgesia & Epidural \\
\hline Diprivan & AstraZeneca & Propofol & Anesthetic & Epidural \\
\hline Exparel & Pacira & Bupivacaine & Analgesia & Epidural \\
\hline \multicolumn{5}{|l|}{ Other } \\
\hline Visudyne & QLT & Verteporfin & $\begin{array}{l}\text { Neovascularization: wet macular } \\
\text { degeneration }\end{array}$ & Intravenous \\
\hline Estrasorb & King & Estrogen & Menopausal therapy & Topical \\
\hline
\end{tabular}

\section{Marketed liposomes}

In 1995, Doxil (PEGylated liposome-encapsulated doxorubicin) became the first liposome drug delivery system approved for human use by the US FDA [144]. Currently, Doxil and other liposomal formulations encapsulating doxorubicin and daunorubicin are widely used to treat ovarian cancer and Kaposi's sarcoma, and to protect patients from anthracycline cardiotoxicity [145]. As shown in Table 5, about 19 liposome and lipid-based drug formulations are approved for human use. Eight are treatments intended for cancers; others are treatments against fungi or microbes, preventive vaccination, analgesia, macular degeneration, and hormone replacement.

Actually, all human clinical trials proposed for product licensing approval by the FDA must be registered with ClinicalTrials.gov, a US Department of Health and Human Services sponsored clinical trial registry. According to ClinicalTrials.gov, there are 1562 interventional drug studies with a liposome platform as of May 2015. Interestingly, no FDA-licensed liposome or lipid nanoparticle is coated with ligands or targeting moieties for homing drug to target tissues, cells or subcellular organelles [3]. Such targeted therapeutics (with or without precise and controlled drug release) are an emerging area of research.

\section{Conclusion}

In the 50 plus years since the concept of their clinical utility was pushed forward by Banghan, liposomes have drawn attention of researchers. Currently, they remain a topical issue, where new preparation methods have been developed as well as new characterization techniques applied. The accumulation of many novel experiences studying the practical aspects of liposomes is reflected in the increasing number of clinical trials involving liposomes. Indeed, with the success of Doxil and other liposomal drug delivery systems, liposomes have already made their transition into the clinic, where they have resulted in improved patient outcomes. This immense challenge was successfully overcome due to suitable stealth degree of liposomes. Application of unmodified nanoparticles are limited by their rapid recognition by macrophages of the RES within a few hours of administration. The inclusion of stealth agents, such as PEG, extend the circulation lifetime of the vehicle. In this way, in contrast to free forms, encapsulated drugs are selectively retained in plasma and are not subject to immediate absorption and filtering by the main organs. Consequently, therapeutic agent would be present at its target in low but stable amounts sufficient to exert an effect.

However, current clinically available liposome-based therapies do not exhibit active targeting at the cellular level and therefore do not benefit from the full potential that nanotechnology can provide. To make a significant therapeutic impact, liposomes must serve as a drug carrier not only to improve drug stability and exposure, but also to enhance the accumulation of a significant amount of drug in the target tissue. Advances in molecular design and chemistry for inclusion of ligand molecules on the liposomal surface improve their interaction with target cells. In the development of ligand-targeted liposomal therapeutics, several considerations should be taken into account. The target antigen should be selectively expressed or overexpressed on the target cells in sufficient quantity so as to ensure firm binding of liposomes to cancer cells. Furthermore, in targeted ligand conjugation, it is crucial to achieve binding of a targeting moiety without losing its functionality after attachment to the liposome.

Future generations of liposomal drug delivery systems are likely to possess multiple and complementary functionalities for stealth, targeting and controlled drug release, and the key topics developed in this review may resolve the current challenges in their development. The continuous study on the design refinement and optimization contribute to the potential of targeting liposomes to 
provide major advances in clinical therapy that could dramatically improve patient outcome.

\section{Acknowledgements}

Eugénia Nogueira (SFRH/BD/81269/2011) holds a scholarship from Fundação para a Ciência e a Tecnologia (FCT). This study was funded by the European Union Seventh Framework Programme (FP7/2007-2013) under grant agreement NMP4-LA2009-228827 NANOFOL. This study was also supported by FEDER through POFC-COMPETE and by national funds from FCT through the project PEst-C/BIA/UI4050/2014 and the strategic funding of ID/BIO/04469/2013 unit. This work was supported by FCT I.P. through the strategic funding UID/BIA/04050/2013.

\section{References}

[1] A.D. Bangham, et al., Negative staining of phospholipids and their structural modification by surface-active agents as observed in the electron microscope, J. Mol. Biol. 8 (1964), 660-IN10.

[2] A. Maheswaran, et al., Liposomal drug delivery systems-a review, Int. J. Pharm. Sci. Rev. Res. 23 (1) (2013) 295-301.

[3] J.C. Kraft, et al., Emerging research and clinical development trends of liposome and lipid nanoparticle drug delivery systems, J. Pharm. Sci. 103 (2014) 29-52.

[4] D.W. Deamer, From banghasomes to liposomes: a memoir of Alec Bangham, 1921-2010, FASEB J. 24 (2010) 1308-1310.

[5] G. Gregoriadis, The carrier potential of liposomes in biology and medicine, N. Engl. J. Med. 295 (1976) 765-770.

[6] T.M. Allen, et al., Liposomal drug delivery systems: from concept to clinical applications, Adv. Drug Delivery Rev. 65 (2013) 36-48.

[7] A.Z. Wang, et al., Nanoparticle delivery of cancer drugs, Annu. Rev. Med. 63 (2012) 185-198.

[8] A. Laouini, et al., Preparation, characterization and applications of liposomes: state of the art, J. Colloid Sci. Biotechnol. 1 (2012) 147-168.

[9] M.-S. Hong, et al., Prolonged blood circulation of methotrexate by modulation of liposomal composition, Drug Delivery 8 (2001) 231-237.

[10] A. Jesorka, et al., Liposomes: technologies and analytical applications, Annu. Rev. Anal. Chem. 1 (2008) 801-832.

[11] M. Uhumwangho, et al., Current trends in the production and biomedical applications of liposomes: a review, J. Med. Biomed. Res. 4 (2005) 9-21.

[12] J. Sabin, et al., Size and stability of liposomes: a possible role of hydration and osmotic forces, Eur. Phys. J. E Soft Matter 20 (2006) 401-408.

[13] D.D. Lasic, Liposomes: from Physics to Applications, Elsevier, 1993, 2015

[14] D.M. Webster, et al., Injectable nanomaterials for drug delivery: carriers, targeting moieties, and therapeutics, Eur. J. Pharm. Biopharm. 84 (2013) $1-20$.

[15] S. Vemuri, et al., Preparation and characterization of liposomes as therapeutic delivery systems: a review, Pharm. Acta Helv. 70 (1995) 95-111.

[16] T. Lian, et al., Trends and developments in liposome drug delivery systems, J. Pharm. Sci. 90 (2001) 667-680.

[17] E. Elizondo, et al., Liposomes and other vesicular systems: structural characteristics, methods of preparation, and use in nanomedicine, Prog. Mol. Biol. Trans. Sci. 104 (2011) 1-52.

[18] G. Gregoriadis, et al., Stability of liposomes in vivo and in vitro is promoted by their cholesterol content and the presence of blood cells, Biochem. Biophys. Res. Commun. 89 (1979) 1287-1293.

[19] A.G. Kohli, et al., Designer lipids for drug delivery: from heads to tails, J Controlled Release 190 (2014) 274-287.

[20] D.C. Drummond, et al., Pharmacokinetics and in vivo drug release rates in liposomal nanocarrier development, J. Pharm. Sci. 97 (2008) 4696-4740.

[21] R.J. Lee, et al., Delivery of liposomes into cultured KB cells via folate receptor-mediated endocytosis, J. Biol. Chem. 269 (1994) 3198-3204.

[22] A.A. Gabizon, Liposome circulation time and tumor targeting: implications for cancer chemotherapy, Adv. Drug Delivery Rev. 16 (1995) 285-294.

[23] T.M. Allen, et al., Uptake of liposomes by cultured mouse bone marrow macrophages: influence of liposome composition and size, BBA, Biomembranes 1061 (1991) 56-64.

[24] D.E. Owens Iii, et al., Opsonization, biodistribution, and pharmacokinetics of polymeric nanoparticles, Int. J. Pharm. 307 (2006) 93-102.

[25] C. Allen, et al., Controlling the physical behavior and biological performance of liposome formulations through use of surface grafted poly(ethylene glycol), Biosci. Rep. 22 (2002) 225-250.

[26] D. Needham, et al., PEG-covered lipid surfaces: bilayers and monolayers, Colloids Surf. B. Biointerfaces 18 (2000) 183-195.

[27] P.L. Ahl, et al., Enhancement of the in vivo circulation lifetime of 1 - $\alpha$-distearoylphosphatidylcholine liposomes: importance of liposomal aggregation versus complement opsonization, BBA, Biomembranes 1329 (1997) 370-382.

[28] M. Vert, et al., Poly(ethylene glycol): protein-repulsive or albumin-compatible? J. Biomater. Sci. Polym. Ed. 11 (2000) 1307-1317.
[29] E. Nogueira, et al., Liposome and protein based stealth nanoparticles, Faraday Discuss 166 (2013) 417-429.

[30] O. Tirosh, et al., Hydration of polyethylene glycol-grafted liposomes, Biophys. J. 74 (1998) 1371-1379.

[31] P.G. de Gennes, Polymers at an interface; a simplified view, Adv. Colloid Interface Sci. 27 (1987) 189-209.

[32] E.T. Dams, et al., Accelerated blood clearance and altered biodistribution of repeated injections of sterically stabilized liposomes, J. Pharmacol. Exp. Ther. 292 (2000) 1071-1079.

[33] T. Ishida, et al., Accelerated blood clearance (ABC) phenomenon upon repeated injection of PEGylated liposomes, Int. J. Pharm. 354 (2008) 56-62.

[34] T. Ishida, et al., Injection of PEGylated liposomes in rats elicits PEG-specific IgM, which is responsible for rapid elimination of a second dose of PEGylated liposomes, J. Control Release 112 (2006) 15-25.

[35] T. Ishida, et al., The contribution of phagocytic activity of liver macrophages to the accelerated blood clearance (ABC) phenomenon of PEGylated liposomes in rats, J. Control Release 126 (2008) 162-165.

[36] A.S. Abu Lila, et al., The accelerated blood clearance (ABC) phenomenon: clinical challenge and approaches to manage, J. Control Release 172 (2013) 38-47.

[37] E. Ambegia, et al., Stabilized plasmid-lipid particles containing PEG-diacylglycerols exhibit extended circulation lifetimes and tumor selective gene expression, Biochim. Biophys. Acta 20 (2005) 155-163.

[38] M.S. Webb, et al., Comparison of different hydrophobic anchors conjugated to poly(ethylene glycol): effects on the pharmacokinetics of liposomal vincristine, Biochim. Biophys. Acta 17 (1998) 272-282.

[39] A. Akbarzadeh, et al., Liposome: classification, preparation, and applications, Nanoscale Res. Lett. 8 (2013) 102

[40] A.D. Bangham, et al., Diffusion of univalent ions across the lamellae of swollen phospholipids, J. Mol. Biol. 13 (1965), 238-IN27.

[41] S.A. Bhai, et al., Liposomes-an overview, J. Pharm. Sci. Innov. (2012) $13-21$.

[42] J.S. Dua, et al., Liposomes: methods of preparation and applications, Int. J. Pharm. Stud. Res. 3 (2012) 14-20.

[43] K. Shashi, et al., A complete review on: liposomes, Int. Res. J. Pharm. 3 (7) (2012) 10-16.

[44] J. Dua, et al., Liposome: methods of preparation and applications, Int. J Pharm. Stud. Res. 3 (2012) 14-20.

[45] E. Mayhew, et al., Characterization of liposomes prepared using a microemulsifier, BBA, Biomembranes 775 (1984) 169-174.

[46] S. Batzri, E.D. Korn, Single bilayer liposomes prepared without sonication, BBA, Biomembranes 298 (1973) 1015-1019.

[47] L. Lesoin, et al., Development of a continuous dense gas process for the production of liposomes, J. Supercrit. Fluids 60 (2011) 51-62.

[48] D.E. Koppel, Analysis of macromolecular polydispersity in intensity correlation spectroscopy: the method of cumulants, J. Chem. Phys. 57 (1972) 4814-4820.

[49] M. Gaumet, et al., Nanoparticles for drug delivery: the need for precision in reporting particle size parameters, Eur. J. Pharm. Biopharm. 69 (2008) $1-9$.

[50] V. Filipe, et al., Critical evaluation of nanoparticle tracking analysis (NTA) by NanoSight for the measurement of nanoparticles and protein aggregates, Pharm. Res. 27 (2010) 796-810.

[51] C. Gardiner, et al., Extracellular vesicle sizing and enumeration by nanoparticle tracking analysis, J. Extracell. Vesicles 2 (2013).

[52] J.M. Freire, et al., Using zeta-potential measurements to quantify peptide partition to lipid membranes, Eur. Biophys. J. 40 (2011) 481-487.

[53] J. Clogston, et al., Zeta potential measurement, in: S.E. McNeil (Ed.), Characterization of Nanoparticles Intended for Drug Delivery, Humana Press, 2011, pp. 63-70.

[54] V. Klang, et al., Electron microscopy of nanoemulsions: an essential tool for characterisation and stability assessment, Micron 43 (2012) 85-103.

[55] V. Klang, et al., Electron microscopy of pharmaceutical systems, Micron 44 (2013) 45-74.

[56] S. Bibi, et al., Microscopy imaging of liposomes: from coverslips to environmental SEM, Int. J. Pharm. 417 (2011) 138-150.

[57] A. Jacquot, et al., Morphological and physical analysis of natural phospholipids-based biomembranes, PLoS One 9 (2014) e107435.

[58] C. Tikshdeep, et al., Liposome drug delivery: a review, Int. J. Pharm. Chem. Sci. 1 (2012) 754

[59] G. Storm, et al., Liposomes: quo vadis? Pharm. Sci. Technol. Today 1 (1998) 19-31.

[60] S. Sharma, et al., Liposomes: vesicular system an overview, Int. J. Pharm. Pharm. Sci. 2 (2010) 11-17.

[61] S. Rane, et al., Influence of liposome composition on paclitaxel entrapment and pH sensitive of liposomes, Int. J. Pharm. Technol. Res. 1 (3) (2009) 914-917.

[62] S. Simoes, et al., On the formulation of pH-sensitive liposomes with long circulation times, Adv. Drug Delivery Rev. 56 (2004) 947-965.

[63] V. Balamuralidhara, et al., pH sensitive drug delivery systems: a review, Am. J. Drug Discovery Dev. 1 (2011) 24-48.

[64] X. Liu, et al., Formation strategies, mechanism of intracellular delivery and potential clinical applications of pH-sensitive liposomes, Asian J. Pharm. Sci. 8 (2013) 319-328

[65] C. Lonez, et al., Cationic liposomal lipids: from gene carriers to cell signaling, Prog. Lipid Res. 47 (2008) 340-347. 
[66] P.L. Felgner, et al., Lipofection: a highly efficient, lipid-mediated DNA-transfection procedure, Proc. Natl. Acad. Sci. U. S. A. 84 (1987) 7413-7417.

[67] W. Li, et al., Lipid-based nanoparticles for nucleic acid delivery, Pharm. Res. 24 (2007) 438-449.

[68] S. Simões, et al., Cationic liposomes for gene delivery, Expert Opin. Drug Delivery 2 (2005) 237-254.

[69] H. Lv, et al., Toxicity of cationic lipids and cationic polymers in gene delivery, J. Controlled Release 114 (2006) 100-109.

[70] R. Lehner, et al., Intelligent nanomaterials for medicine: carrier platforms and targeting strategies in the context of clinical application, Nanomed. Nanotechnol. Biol. Med. 9 (2013) 742-757.

[71] R. Sawant, et al., Challenges in development of targeted liposomal therapeutics, AAPS J. 14 (2012) 303-315.

[72] J.L. Markman, et al., Nanomedicine therapeutic approaches to overcome cancer drug resistance, Adv. Drug Delivery Rev. 65 (2013) 1866-1879.

[73] G.T. Noble, et al., Ligand-targeted liposome design: challenges and fundamental considerations, Trends Biotechnol. 32 (2014) 32-45.

[74] J.S. Poovassery, et al., Antibody targeting of HER2/HER3 signaling overcomes heregulin-induced resistance to PI3K inhibition in prostate cancer, Int. J. Cancer 4 (2014), 29378.

[75] G.D. Lewis Phillips, et al., Targeting HER2-positive breast cancer with trastuzumab-DM1, an antibody-cytotoxic drug conjugate, Cancer Res. 68 (2008) 9280-9290.

[76] B.K. Ko, et al., Combination of novel HER2-targeting antibody 1 E11 with trastuzumab shows synergistic antitumor activity in HER2-positive gastric cancer, Mol. Oncol. 28 (2014) 00231-00232.

[77] S. Brack, et al., A bispecific HER2-targeting FynomAb with superior antitumor activity and novel mode of action, Mol. Cancer Ther. 13 (2014) 2030-2039

[78] J. Albanell, et al., Mechanism of action of anti-HER2 monoclonal antibodies: scientific update on trastuzumab and 2C4, Adv. Exp. Med. Biol. 532 (2003) 253-268.

[79] W. Xie, et al., Generation and characterization of a novel human IgG1 antibody against vascular endothelial growth factor receptor 2, Cancer Immunol. Immunother. 63 (2014) 877-888.

[80] H.J. Choi, et al., A heterodimeric Fc-based bispecific antibody simultaneously targeting VEGFR-2 and Met exhibits potent antitumor activity, Mol. Cancer Ther. 12 (2013) 2748-2759.

[81] A.X. Zhu, et al., A phase II and biomarker study of ramucirumab, a human monoclonal antibody targeting the VEGF receptor-2, as first-line monotherapy in patients with advanced hepatocellular cancer, Clin. Cancer Res. 19 (2013) 6614-6623.

[82] D. Hoshina, et al., Establishment of a novel experimental model of human angiosarcoma and a VEGF-targeting therapeutic experiment, J. Dermatol Sci. 70 (2013) 116-122.

[83] V.P. Chekhonin, et al., VEGF in tumor progression and targeted therapy, Curr. Cancer Drug Targets 13 (2013) 423-443.

[84] A.P. Mann, et al., Identification of thioaptamer ligand against E-selectin: potential application for inflamed vasculature targeting, PLoS One 5 (2010), 13050

[85] R. Faryammanesh, et al., SDA, a DNA aptamer inhibiting E- and P-selectin mediated adhesion of cancer and leukemia cells, the first and pivotal step in transendothelial migration during metastasis formation, PLoS One 9 (2014) e93173.

[86] L. Li, et al., Nucleolin-targeting liposomes guided by aptamer AS1411 for the delivery of siRNA for the treatment of malignant melanomas, Biomaterials 35 (2014) 3840-3850.

[87] W. Hwang do, et al., A nucleolin-targeted multimodal nanoparticle imaging probe for tracking cancer cells using an aptamer, J. Nucl. Med. 51 (2010) 98-105.

[88] S. Soundararajan, et al., The nucleolin targeting aptamer AS1411 destabilizes Bcl-2 messenger RNA in human breast cancer cells, Cancer Res. 68 (2008) 2358-2365.

[89] W. Zhou, et al., Aptamer-nanoparticle bioconjugates enhance intracellular delivery of vinorelbine to breast cancer cells, J. Drug Target. 22 (2014) 57-66.

[90] J. Guo, et al., Aptamer-functionalized PEG-PLGA nanoparticles for enhanced anti-glioma drug delivery, Biomaterials 32 (2011) 8010-8020.

[91] H. Qi, et al., Transferrin-targeted magnetic/fluorescence micelles as a specific bi-functional nanoprobe for imaging liver tumor, Nanoscale Res. Lett. 9 (2014) 9-595.

[92] X. Wang, et al., Targeted delivery of tumor suppressor microRNA-1 by transferrin-conjugated lipopolyplex nanoparticles to patient-derived glioblastoma stem cells, Curr. Pharm. Biotechnol. 15 (2014) 839-846.

[93] L. Qin, et al., A dual-targeting liposome conjugated with transferrin and arginine-glycine-aspartic acid peptide for glioma-targeting therapy, Oncol. Lett. 8 (2014) 2000-2006.

[94] S. Tortorella, et al., Transferrin receptor-mediated endocytosis: a useful target for cancer therapy, J. Membr. Biol. 247 (2014) 291-307.

[95] T.R. Daniels, et al., The transferrin receptor and the targeted delivery of therapeutic agents against cancer, Biochim. Biophys. Acta 3 (2012) 291-317.

[96] Z.M. Qian, et al., Targeted drug delivery via the transferrin receptor-mediated endocytosis pathway, Pharmacol. Rev. 54 (2002) 561-587.

[97] H. Li, et al., Transferrin/transferrin receptor-mediated drug delivery, Med. Res. Rev. 22 (2002) 225-250
[98] H. Li, et al., The role of the transferrin-transferrin-receptor system in drug delivery and targeting, Trends Pharmacol. Sci. 23 (2002) 206-209.

[99] T.R. Daniels, et al., The transferrin receptor part I: biology and targeting with cytotoxic antibodies for the treatment of cancer, Clin. Immunol. 121 (2006) $144-158$

[100] T.R. Daniels, et al., The transferrin receptor part II: targeted delivery of therapeutic agents into cancer cells, Clin. Immunol. 121 (2006) 159-176.

[101] R. Nedaeinia, et al., EGFR as a potential target for the treatment of pancreatic cancer: dilemma and controversies, Curr. Drug Targets 25 (2014) 25.

[102] J. Mendelsohn, et al., Epidermal growth factor receptor targeting in cancer, Semin. Oncol. 33 (2006) 369-385

[103] J. Baselga, et al., Critical update and emerging trends in epidermal growth factor receptor targeting in cancer, J. Clin. Oncol. 23 (2005) 2445-2459

[104] P.M. Harari, Epidermal growth factor receptor inhibition strategies in oncology, Endocrine-Relat. Cancer 11 (2004) 689-708.

[105] C. Yewale, et al., Epidermal growth factor receptor targeting in cancer: a review of trends and strategies, Biomaterials 34 (2013) 8690-8707.

[106] X. He, et al., RGD peptide-modified multifunctional dendrimer platform fo drug encapsulation and targeted inhibition of cancer cells, Colloids Surf. B. Biointerfaces 11 (2014) 82-89.

[107] A. Raj, et al., Binding and uptake of novel RGD micelles to the alphavbeta3 integrin receptor for targeted drug delivery, J. Drug Target. 22 (2014) 518-527.

[108] N.M. Javali, et al., Fatty acid-RGD peptide amphiphile micelles as potential paclitaxel delivery carriers to alpha(v) beta(3) integrin overexpressing tumors, Pharm. Res. 29 (2012) 3347-3361.

[109] S.I. Shen, et al., Synthesis and characterization of RGD-fatty acid amphiphilic micelles as targeted delivery carriers for anticancer agents, J. Drug Target. 15 (2007) 51-58

[110] L.L. Cai, et al., RGD peptide-mediated chitosan-based polymeric micelles targeting delivery for integrin-overexpressing tumor cells, Int. J. Nanomed. 6 (2011) 3499-3508.

[111] A. Accardo, et al., Octreotide labeled aggregates containing platinum complexes as nanovectors for drug delivery, J. Pept. Sci. 19 (2013) 190-197

[112] J. Zhang, et al., A novel octreotide modified lipid vesicle improved the anticancer efficacy of doxorubicin in somatostatin receptor 2 positive tumor models, Mol. Pharm. 7 (2010) 1159-1168.

[113] M. Sun, et al., Octreotide-modification enhances the delivery and targeting of doxorubicin-loaded liposomes to somatostatin receptors expressing tumor in vitro and in vivo, Nanotechnology 21 (2010) 0957-4484.

[114] M. Huo, et al., Somatostatin receptor-mediated tumor-targeting drug delivery using octreotide-PEG-deoxycholic acid conjugate-modified $\mathrm{N}$-deoxycholic acid-O,N-hydroxyethylation chitosan micelles, Biomaterials 33 (2012) 6393-6407.

[115] A. Zou, et al., Octreotide-modified $N$-octyl-O,N-carboxymethyl chitosan micelles as potential carriers for targeted antitumor drug delivery, J. Pharm. Sci. 101 (2012) 627-640.

[116] Y. Li, et al., Targeted delivery of macromolecular drugs: asialoglycoprotein receptor (ASGPR) expression by selected hepatoma cell lines used in antiviral drug development, Curr. Drug Delivery 5 (2008) 299-302.

[117] F. Zhang, et al., Hepatic-targeting microcapsules construction by self-assembly of bioactive galactose-branched polyelectrolyte for controlled drug release system, J. Colloid Interface Sci. 317 (2008) 477-484.

[118] F. Zhang, et al., Thermal treatment of galactose-branched polyelectrolyte microcapsules to improve drug delivery with reserved targetability, Int. J. Pharm. 357 (2008) 22-31.

[119] G. Huang, et al., Asialoglycoprotein receptor-targeted superparamagnetic iron oxide nanoparticles, Int. J. Pharm. 360 (2008) 197-203.

[120] J. Wu, et al., Targeting hepatocytes for drug and gene delivery: emerging novel approaches and applications, Front. Biosci. 1 (2002) d717-d725.

[121] Y.G. Assaraf, et al., The folate receptor as a rational therapeutic target for personalized cancer treatment, Drug Resist. Updates 17 (2014) 89-95.

[122] P.P. Peethambaram, et al., A phase I pharmacokinetic and safety analysis of epothilone folate (BMS-753493), a folate receptor targeted chemotherapeutic agent in humans with advanced solid tumors, Invest. New Drugs 8 (2014) 8.

[123] K.D. Lee, et al., Self-organized nanoparticles based on chitosan-folic acid and dextran succinate-doxorubicin conjugates for drug targeting, Arch. Pharmacal. Res. 37 (2014) 1546-1553.

[124] V.M. Gaspar, et al., Folate-targeted multifunctional amino acid-chitosan nanoparticles for improved cancer therapy, Pharm. Res. 4 (2014) 4.

[125] C. Su, et al., Carboxymethyl-beta-cyclodextrin conjugated nanoparticles facilitate therapy for folate receptor-positive tumor with the mediation of folic acid, Int. J. Pharm. 474 (2014) 202-211.

[126] Y. Li, et al., Therapeutic effect of folate-targeted and PEGylated phytosome loaded with a mitomycin C-soybean phosphatidyhlcholine complex. Mol. Pharm. 11 (2014) 3017-3026.

[127] G. Wu, et al., Folate-modified doxorubicin-loaded nanoparticles for tumor-targeted therapy, Pharm. Biol. 52 (2014) 978-982.

[128] P. Yu, et al., Folic acid-conjugated iron oxide porous nanorods loaded with doxorubicin for targeted drug delivery, Colloids Surf. B. Biointerfaces 120 (2014) 142-151.

[129] Y. Zhang, et al., Folate-targeted paclitaxel-conjugated polymeric micelles inhibits pulmonary metastatic hepatoma in experimental murine $\mathrm{H} 22$ metastasis models, Int. J. Nanomed. 9 (2014) 2019-2030. 
[130] F. Wang, et al., Folate-mediated targeted and intracellular delivery of paclitaxel using a novel deoxycholic acid-O-carboxymethylated chitosan-folic acid micelles, Int. J. Nanomed. 7 (2012) 325-337.

[131] M.K. Yu, et al., Targeting strategies for multifunctional nanoparticles in cancer imaging and therapy, Theranostics 2 (1) (2012) 3-44.

[132] P. Marqués-Gallego, et al., Ligation Strategies for Targeting Liposomal Nanocarriers, BioMed Research International, 2014.

[133] J. Conde, et al., Revisiting 30 years of biofunctionalization and surface chemistry of inorganic nanoparticles for nanomedicine, Front. Chem. 2 (2014) 48 .

[134] T. Steenpaß, et al., Tresylated PEG-sterols for coupling of proteins to preformed plain or PEGylated liposomes, BBA, Biomembranes 1758 (2006) 20-28.

[135] X. Zhao, et al., Targeted drug delivery via folate receptors, Expert Opin. Drug Delivery 5 (2008) 309-319.

[136] A.R. Hilgenbrink, et al., Folate receptor-mediated drug targeting: from therapeutics to diagnostics, J. Pharm. Sci. 94 (2005) 2135-2146.

[137] C.P. Leamon, et al., Exploitation of the folate receptor in the management of cancer and inflammatory disease, Vitam. Horm. 79 (2008) 203-233.
[138] A. Gabizon, et al., Tumor cell targeting of liposome-entrapped drugs with phospholipid-anchored folic acid-PEG conjugates, Adv. Drug Delivery Rev. 56 (2004) 1177-1192.

[139] Y. Liu, et al., Synthesis and evaluation of a novel lipophilic folate receptor targeting ligand, Anticancer Res. 31 (2011) 1521-1525.

[140] G. Xiang, et al., Synthesis and evaluation of a novel ligand for folate-mediated targeting liposomes, Int. J. Pharm. 356 (2008) 29-36.

[141] S. Xiong, et al., Preparation, therapeutic efficacy and intratumoral localization of targeted daunorubicin liposomes conjugating folate-PEG-CHEMS, Biomed. Pharmacother. 65 (2011) 2-8.

[142] A. Verma, et al., Effect of surface properties on nanoparticle-cell interactions, Small 6 (2010) 12-21.

[143] A. Albanese, et al., The effect of nanoparticle size, shape, and surface chemistry on biological systems, Annu. Rev. Biomed. Eng. 14 (2012)1-16.

[144] N.D. James, et al., Liposomal doxorubicin (Doxil): an effective new treatment for Kaposi's sarcoma in AIDS, Clin. Oncol. (R. Coll. Radiol.) 6 (5) (1994) 294-296.

[145] T. Safra, et al., Pegylated liposomal doxorubicin (Doxil): reduced clinical cardiotoxicity in patients reaching or exceeding cumulative doses of $500 \mathrm{mg} / \mathrm{m}^{2}$, Ann. Oncol. 11 (2000) 1029-1033. 